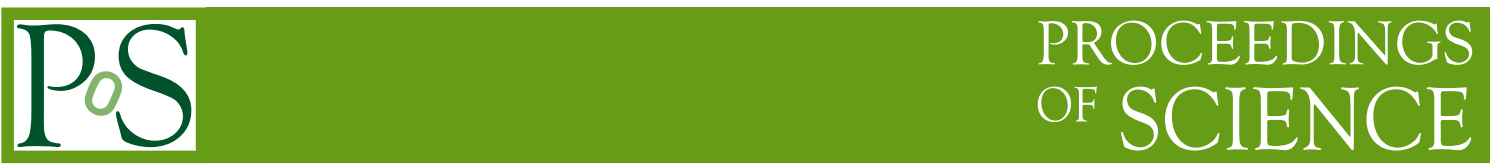

\title{
Extreme-value analysis of the X-ray emission of Cygnus X-1
}

\author{
Maria Süveges* \\ ISDC Data Centre for Astrophysics, Astronomical Observatory of Geneva, Geneva University \\ E-mail: Maria.Suveges@unige.ch
}

\section{Stéphane Paltani}

ISDC Data Centre for Astrophysics, Astronomical Observatory of Geneva, Geneva University

E-mail: Stephane.Paltanieunige.ch

\begin{abstract}
The multiplicative model of Uttley et al. (2005) for the X-ray emission from X-ray binaries accounts well for the observed global characteristics of the time series such as the linear flux-rms relationship and the lognormal distribution of the fluxes. However, models that are fitted to the bulk of the data may not provide satisfactory models for the extreme events in the series: these might be produced by other unrecognized processes. As the model of Uttley et al. (2005) implies a well-determined structure of events above high thresholds, we applied methods from extremevalue statistics to check whether the same process can be responsible for the extremes in the data. We first considered the limiting distribution of large events as expected from the model, and compared to that of a model fitted to data from Cygnus X-1. Next, the clustering characteristics of the observed large events was compared to what is expected. Both comparisons show that the multiplicative model can indeed generate the observed extreme events, though there are indications of nonstationarity in the baseline level of the process and also of short periods where the observed extreme-value characteristics could be incompatible with the model implications.
\end{abstract}

Fast $X$-ray timing and spectroscopy at extreme count rates

February 7-11, 2011

Champéry, Switzerland

${ }^{*}$ Speaker. 


\section{Introduction}

Time-domain methods are only rarely applied in the analysis of astrophysical time series for $\mathrm{X}$-ray binaries. Their X-ray variability is usually modelled in frequency space, by the means of the power spectral density (PSD). However, the form of the PSD is not stable over time even for one source in the same state, whereas a lognormal distribution of the fluxes and a linear relationship between the mean flux and the root-mean-square variability on a wide range of time-scales have already been observed both in X-ray binaries and narrow-line Seyfert 1 AGN [1,2,3,4,5]. As such, they might pertain to processes common over a broad family of objects, and may be fundamental features of accreting systems.

To explain the PSD shape, there were several competing models proposed in the literature: for instance, shot-noise models [6], thundercloud models [7] and self-organized criticality (SOC) $[8,9]$. The multiplicative model of [10] is one model that is intrinsically able to reproduce both the lognormal univariate marginal distribution of the fluxes and the linear flux-rms relationship. The thundercloud model is also able to yield a linear rms-flux relationship, but it implies a power law tail instead of a lognormal one. Additive shot-noise models, though lognormality can be obtained by supposing particular shot width and size distributions, fail to automatically produce the rmsflux relationship, just as SOC models. Thus, the multiplicative model, backed up by a possible background physical mechanism proposed by [11], is unique in the sense that there is no finetuning required to reproduce these broad characteristics of the X-ray variability.

We checked the ability of this model also for the generation of the occurrence of very large fluxes. Instead of counting events in a time series which was simulated based on rough approximation of the PSD structure, we used well-developed tools from extreme-value statistics which have been regularly applied for risk estimation in climatology, finance or engineering for decades $[12,13,14,15]$. Each of the models listed above has particular extreme-flux distribution properties, and the general performance or a possible contribution of each can be investigated by carefully examining the statistical characteristics of the extreme events of a time series. Heavy-tailed or additive lognormal models can be distinguished from the multiplicative model by their differing dependence structure and their different extreme-value distributions. In Section 2, we present briefly the formulation of the multiplicative model, and summarize the extreme-value methods that are novel in astrophysics. Then, in Section 3, we apply these methods on the same data set as in [10], a time series of Cygnus X-1 in the hard state observed by RXTE. Section 4 gives a short summary of the results.

\section{Statistical methods}

\subsection{Multiplicative model}

According to [10], an adequate model that reproduces the features observed in the light curve of Cyg X-1 in the hard state is

$$
Y_{t} \sim \exp \left(X_{t}\right)+\tau
$$


where $\tau$ is a constant background, and $X_{t}$ is a Gaussian linear process. Then the observed fluxes $Y_{t}$, apart from an additional Poissonization, follow the shifted lognormal distribution

$$
f(x ; \tau, \mu, \sigma)=\frac{1}{\sigma \sqrt{2 \pi}(x-\tau)} \exp \left\{-\frac{[\log (x-\tau)-\mu]^{2}}{2 \sigma^{2}}\right\} .
$$

Linear Gaussian (ARMA) models can be used as approximate models for a very broad class of processes: for any stationary process with autocovariance function $\gamma(h)$ such that $\lim _{h \rightarrow \infty} \gamma(h)=0$, and for any integer $k$, there can be found an ARMA process with autocovariance function $\gamma_{X}(h)$ such that for $h=1, \ldots, k, \gamma(h)=\gamma_{X}(h)$ [16]. The existence of an approximate Gaussian linear process with the same autocorrelation structure is therefore assured if Gaussianity is assured on the log scale. This means that a fit, which is in a statistical sense satisfactory, does not necessarily have any physical meaning. After fitting the background shift $\tau_{t}$ possibly with time-dependence by maximum likelihood, and after taking $\log \left(Y_{t}-\tau_{t}\right)$, we may expect to find a sufficiently well-fitting ARMA model for a stationary interval of the data, but physical interpretation of the obtained model should be considered very carefully.

\subsection{Extreme-value analysis}

In extreme-value statistics, an exceedance is defined as an observation $X$ that exceeds a high threshold $u$, where $u$ is chosen usually so that only a low $(<5 \%)$ percent of the data is exceedance. An excess $X-u$ from a random sample having a common distribution $F$ follows approximately

$$
H(y)= \begin{cases}1-(1+\xi y / \psi)^{-1 / \xi}, & \xi \neq 0 \\ 1-\exp (-y / \psi), & \xi=0\end{cases}
$$

for $\psi>0, y>0$ under mild conditions that are satisfied by all practically interesting continuous distribution functions $F[13,14]$. (2.2) is called the Generalized Pareto distribution (GPD). The shape parameter $\xi$ determines the tail decay and the probability of the occurrence of very large observations; the scale parameter $\psi$ the spread of the distribution, similarly to a variance parameter. The central importance of the GPD is the fact that it behaves like the normal distribution in the Central Limit Theorem. Regardless of the original distribution $F$ of the sample, under broad conditions, the sample mean follows asymptotically a normal distribution due to the Central Limit Theorem. Likewise, under different but still broad conditions, the excesses of a very high threshold of the same sample follow asymptotically a GPD.

However, the GPD class comprises three differently behaved types according to the sign of the shape parameter $\xi$. In the case $\xi<0$, there is an upper boundary of the distribution of the excesses. In the case $\xi=0$, there is no finite upper endpoint for the excesses, but the tail probabilities decrease quickly. This is the limiting distribution of excesses from exponential-tailed distributions like the normal, the exponential, the gamma or the lognormal distribution. Finally, the case $\xi>0$ describes the excesses of distributions with power law tail. In this case, the probability of observing a really large extreme value is more substantial than for exponential tail.

The GPD is the asymptotic limiting distribution of the excesses from not only independent sequences, but stationary dependent ones too, under conditions that hold for most practically interesting processes. Dependence nevertheless has several consequences, one of which is the clustering 
of extremes: an independent sequence of random variables will have excesses arriving individually, whereas a dependent one will likely have excesses close-by. The number of extremes close together depends on the strength of dependence. This effect is statistically formulated by the parameter $\theta$ called the extremal index, which is equal to the inverse average extreme cluster size; for instance, in a process that produces extremes on average in pairs, $\theta=1 / 2$, and for an independent sequence, $\theta=1$. On the other hand, even dependent processes can have $\theta=1$, and therefore extremal behaviour identical to an independent process; an example of this is the family of Gaussian linear processes, which is in the background of the multiplicative model. Estimation of the extremal index is possible by fitting a mixture of exponential and a point mass distribution with $\theta$ as parameter to the time intervals between exceedances $[17,18]$.

In summary, if the extreme X-ray emission in the low/hard state of Cygnus X-1 can be adequately modelled by the multiplicative model, we expect to see from extreme-value fits a shape parameter equal to 0 corresponding to the lognormal univariate distribution, and an average cluster size around 1, corresponding to the linear Gaussian time series structure in the background. Values deviating from these would hint at contributions from other processes. A positive shape parameter could suggest the additional presence of a thundercloud model-like or a SOC-like process. A zero shape parameter combined with an extremal index significantly below 1 may hint at dominant additive contributions from only a small number of regions, each generating lognormally distributed fluxes.

\section{Data analysis}

Our data set was an RXTE time series of Cygnus X-1 observed on December 16-19, 1996, in the energy band $2-13 \mathrm{keV}$, binned into $0.125 \mathrm{~s}$ bins. It consists of 9 continuous periods; the source remained in the low/hard state, with an approximately stable PSD. We analyzed each of the 9 periods, obtaining very similar results on all.

Mean. Stationarity is a prerequisite for the validity of the multiplicative model. In order to check it, we first estimated the mean of each continuous observational period with a kernel smoothing method (see e.g. [19], Ch.7, Nadaraya-Watson estimator) with two different bandwidths of 450s and 100s. Then we resampled the observations in each period with equal probability and with replacement 200 times to obtain 200 simulated time series of the same length as the original, thereby breaking any systematic pattern of slowly varying mean, and repeated the Nadaraya-Watson estimation on each repetition. A confidence band can be constructed with pointwise significance level of 0.05 , by taking the 0.025 - and 0.975 -quantiles of the 200 simulated smooth values at each time point, and a constant mean can be accepted if the estimated mean of the original time series remains within the band. The results on all periods excluded the hypothesis of constant mean: the curve of the smooth estimate of the mean leaves the bootstrap confidence band in all 9 periods (the result for period 9 is shown in the left panel of Figure 1). Similar smooth estimates of the high quantiles show variations parallel to the mean (the smooth 0.97 quantile is shown for period 9 in the right panel of Figure 1, superposed on the observations).

Extreme-value analysis. To account for this nonstationarity in the data, we must use sliding window estimates for the extreme value analysis too. We accepted approximate stationarity in windows of $450 \mathrm{~s}$, and applied the following procedure: (1) Among the smooth high quantiles, 

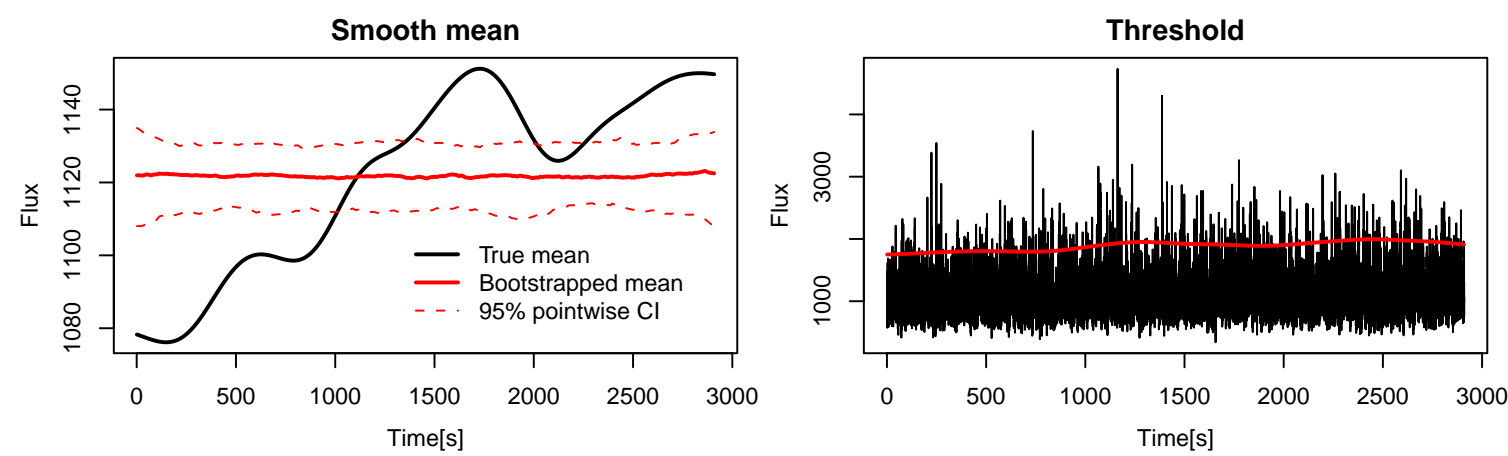

Figure 1: Smooth mean (left) and smooth 0.97-quantile (right) for a continuous segment of Cygnus X-1 fluxes.

we selected one as threshold for each of the 9 periods by checking the quality of extreme-value models, and calculated the sample of excesses by subtracting the local value of the threshold from the observations. (2) We estimated the local shape and scale parameters $\xi(t)$ and $\psi(t)$ from a GPD model by a maximum likelihood fit in sliding windows of the same length. (3) We estimated the local extremal index $\theta(t)$ from an exponential-point mass model by a maximum likelihood fit in sliding windows of the same length.
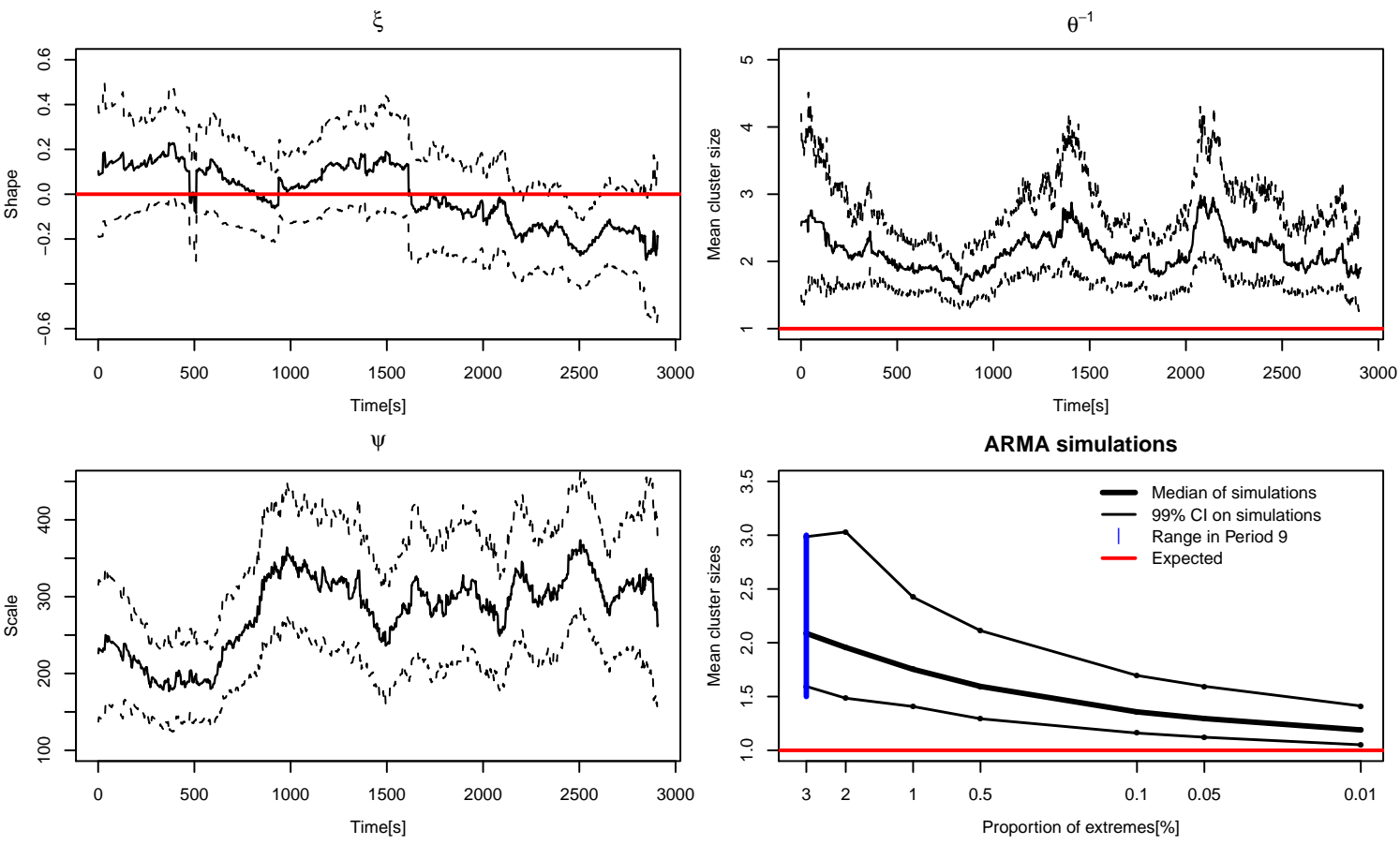

Figure 2: Sliding window shape (upper left), scale (lower left) and average cluster size (upper right) estimates for a continuous segment of Cygnus X-1 fluxes, and the average cluster size depending on thresholds in simulated ARMA processes (lower right). Estimates are plotted with solid lines, 95\% pointwise confidence intervals with dashed lines.

The results of the fits are the following:

- The estimated time-varying shape parameter $\hat{\xi}(t)$ is almost everywhere compatible with the value 0 . There are only very short periods where 0 is outside the pointwise confidence inter- 
val, implying a significant local deviation from the model. In most of these short intervals $\hat{\xi}(t)<0$, excluding a transitory contribution from any process that generates power lawtailed fluxes (e.g. SOC models). However, as these confidence intervals are pointwise, and they represent the results of many local tests, we can expect several excursions even under the hypothesis of constant shape parameter, and the deviations are overall nonsignificant. Nevertheless, the longest of the deviating intervals exhibits a systematic decrease of the shape parameter, presented in the left upper panel of Figure 2. Unfortunately, it occurs at the end of period 9, and the end of the observational period does not allow to follow the further development of the source.

- The estimated mean cluster size, $1 / \hat{\theta}(t)$ and its confidence intervals (upper right panel, Figure 2 only for period 9) seem to exclude the multiplicative process. However, for time series with linear Gaussian structure in the background, very high thresholds should be used [13]. Such high thresholds are not attainable for our data, partly because of the breaks between observational periods, but mainly because nonstationarity requires us to use short windows. Therefore, we checked the plausibility of the multiplicative model by simulation. First, we estimated a smooth shift parameter $\tau_{t}$ from the fitting of the shifted lognormal distribution. Then to the time series $\log \left(Y_{t}-\tau_{t}\right)$, we fitted Gaussian ARMA models using the same sliding window techniques, and estimated their parameters. Finally, we generated 1000 stationary ARMA sequences with a few selected parameter sets, each time long enough so that the use of higher quantiles gave the same number of exceedances as in our short sliding windows, and checked $1 / \hat{\theta}(t)$ in the simulations. The result is shown in the bottom right panel of Figure 2. The slow convergence of the average cluster size to 1 can also be observed, thus confirming the compatibility of the exponentiated Gaussian linear process with the observations.

\section{Discussion}

We checked the predictions of the multiplicative model of [10] on the extreme events of the Cygnus X-1 in its low/hard state in detail. Applying methods taken from extreme-value statistics, we confirmed that the multiplicative model is compatible with the observed extreme structure, and no evidence for contribution from additional processes was found. However, a contribution from an additive process with lognormal margins cannot be excluded, since the only discriminative parameter, the extremal index, cannot be reliably checked because of nonstationarity. A decrease of the shape parameter towards negative values is possible at the end of the time series, which hints at finite-tailed processes. As all models in the literature predict exponential- or power law-tailed processes, further investigations are necessary to confirm or exclude the existence of such periods.

\section{References}

[1] P. Uttley and I. M. McHardy, The flux-dependent amplitude of broadband noise variability in X-ray binaries and active galaxies, MNRAS 323 (2001) L26 -L30

[2] S. Vaughan, A. C. Fabian and K. Nandra, X-ray continuum variability of MCG-6-30-15, MNRAS 339 (2003) 1237-1255 
[3] S. Vaughan, R. Edelson, R. S. Warwick and P. Uttley, On characterizing the variability properties of $X$-ray light curves from active galaxies, MNRAS 345 (2003) 1271-1284

[4] P. Uttley, SAX J1808.4-3658 and the origin of X-ray variability in X-ray binaries and active galactic nuclei, MNRAS 347 (2005) L61-L65

[5] I. M. McHardy, I. E. Papadakis, P. Uttley, M. J. Page and K. O. Mason, Combined long and short time-scale X-ray variability of NGC 4051 with RXTE and XMM-Newton, MNRAS 348 (2004) 783-801

[6] H. Lehto, A model for 1/f-type variability in active galactic nuclei, in proceedings Two Topics in X-Ray Astronomy (1989) 499-503

[7] A. Merloni and A. C. Fabian, Thunderclouds and accretion discs: a model for the spectral and temporal variability of Seyfert 1 galaxies, MNRAS 328 (2001) 958-968 [astro-ph/ 0104271 ]

[8] S. Mineshige, N. B. Ouchi and H. Nishimori, On the generation of 1/f fluctuations in X-rays from black-hole objects, PASJ 46 (1994) 97-105

[9] M. Takeuchi, S. Mineshige and H. Negoro, X-Ray Fluctuations from Black-Hole Objects and Self Organization of Accretion Disks, PASJ 47 (1995) 617-627

[10] P. Uttley, I. M. McHardy and S. Vaughan, Non-linear X-ray variability in X-ray binaries and active galaxies, MNRAS 359 (2005) 345-362 [astro-ph/ 0502112 ]

[11] A. Ingram, C. Done and P. C. Fragile, Low-frequency quasi-periodic oscillations spectra and Lense-Thirring precession, MNRAS 397 (2009) L101-105

[12] P. Embrechts, C. Klüppelberg and T. Mikosch, Modeling Extremal Events for Insurance and Finance, Springer-Verlag, Berlin, 1997

[13] M. R. Leadbetter, G. Lindgren and H. Rootzén, Extremes and Related Properties of Random Sequences and Processes, Springer-Verlag, New York, 1983

[14] S. G. Coles, An Introduction to Statistical Modeling of Extreme Values, Springer-Verlag, London, 2001

[15] B. Finkenstadt and H. Rootzén (eds.), Extreme Values in Finance, Telecommunications, and the Environment, Chapman and Hall, 2003

[16] P. J. Brockwell and R. A. Davis, Time series: Theory and Methods, Springer Science+Business Media, 2006

[17] C. A. T. Ferro and J. Segers, Inference for clusters of extreme values, Journal of the Royal Statistical Society, Series B 65 (2003) 545-556

[18] M. Süveges and A. C. Davison, Model misspecification in peaks over threshold analysis, Annals of Applied Statistics 4 (2010) 203-221

[19] A. C. Davison, Statistical Models, Cambridge University Press, Cambridge, 2003 